\title{
SUMMARY/PROGRESS REPORT
}

\author{
Project Title:
}

\section{Permanganate Treatment of DNAPLs in Reactive Barriers and Source Zone Flooding Schemes}

DOE Report Number (for DOE Labs only, if available): $\mathbf{7 3 7 4 5}$

Grant Number (if applicable): FG07-00ER15115

Publication Date: November 8, 2001

Lead Principal Investigator:

Name: Dr. Frank W. Schwartz

Institution: Ohio State University

Address: 125 S. Oval Mall, Columbus, OH 43210, USA

Phone Number: (614) 292-6196

E-mail address: frank@geology.ohio-state.edu

Co-Principal Investigator:

Name: Dr. Eung Seok Lee (Postdoctoral Researcher)

Institution: Ohio State University

Address: 125 S. Oval Mall, Columbus, $\mathrm{OH} 43210$, USA

Phone Number: (614) 292-0585

E-mail address: elee@geology.ohio-state.edu

Graduate Students

X. David Li

Y. C. Fang

\section{PROGRESS REPORT}

\section{Research Objective}

We are conducting basic research on a new delivery scheme that involves mixing the appropriate quantity of $\mathrm{KMnO}_{4}$ as a solid into the zone of DNAPL contamination. This scheme takes advantage of the tendency for reaction products to reduce the permeability of the treatment zone, which will control the dissolution of the solid and keep the $\mathrm{MnO}_{4}^{-}$in contact with the DNAPL. What needs to be determined, however, is how various solid forms of $\mathrm{KMnO}_{4}$ behave in porous media and dissolve under the combined influence of fluid flow and $\mathrm{MnO}_{2}$ precipitation.

The goals of this study are to (1) to elucidate the dissolution/reaction processes accompanying the amendment of media containing chlorinated solvents with $\mathrm{KMnO} 4$ and (2) to assess the potential for solid $\mathrm{KMnO}_{4}$ amendments as a scheme to clean up source zones. This research involves a combined experimental/modeling study that builds on our previous DOE-sponsored work in understanding of how $\mathrm{KMnO}_{4}$ can be used with in situ cleanups of contaminated sites. The specific objectives of this study are (1) to describe through column experiments how solid forms of potassium permanganate $\left(\mathrm{KMnO}_{4}\right)$ behave in saturated media with and without TCE and PCE. (2) to undertake flow tank studies that examine the hydraulic impact of reaction products (especially $\mathrm{MnO}_{2}$ ) on the flux of water through the zone of contamination, and (3) to represent the process understanding in flow and transport models that demonstrate the potential applicability of the approach. 


\section{Research Progress and Implications}

As of the first year of a 3-year project, we have one post-doc researcher and two $\mathrm{Ph}$. $\mathrm{D}$. graduate students working on different aspects of the project. A series batch experiment, 2-D flow tank experiment have been conducted to investigate the problems related to the delivery of the permanganate to the subsurface contaminated area, and try to eliminate the negative impact caused by the manganese oxide precipitation. Using 3-D flow tank experiments, a subsurface monitoring system was tested and results shown promising future. Primary results of this study have been presented in the national conferences and as papers in the peer-reviewed publications.

(1) Permanganate reactive barrier system: In order to effectively deliver permanganate solid to the subsurface contaminated area, a permanganate reactive barrier system (PRBS) was designed. The design includes a series vertical wells to deliver solid potassium permanganate into the subsurface, through diffusion out of the well casing. The idea is to form a zone of localized reaction through dispersive mixing with dissolved TCE. Thus, as the contaminant plume passes the PRBS, in situ oxidation will destroy the contaminant and prevent further spreading.

A 2-D flow tank experiment was conducted to test the efficacy of PRBS for more than four weeks. The results indicate that the system is capable of delivering the permanganate at a relative stable rate to the plume. The design works well in reducing the concentration of the TCE contaminant plume. The measured chloride concentration at the outlet also indicates the break down of TCE with time. From the visual observation, we saw that the manganese dioxide precipitated on the down flow side of the PRBS and accumulated with time. The proof-of-concept experiment demonstrated how the PRBS could able to delivery permanganate to the subsurface. At the same time, the permeability related pore plugging caused by the manganese oxide precipitates is still a potential problem for long term application. The result of this study has been presented at the American Chemical Society (ACS) annul meeting, and a paper was accepted by ACS and is now in press ( $\mathrm{Li}$ and Schwartz, 2001)

(2) Manganese oxide dissolution experiments: Experiments have been carried out to test the dissolution of the manganese oxide in the present of several chemical additions. The results have shown that the dissolution rate has increased dramatically with the present of citric acid, oxalic acid, and ethylenedinitrilotetraacetic acid (EDTA).

A thin, small 2-D glass flow tank with Teflon end fittings was constructed for the experiment. The flow tank was filled with medium silica sand (US Silica, Ottawa, IL) embedded with fine glass beads lens, which act as low permeability zone. 2 gram of the blue dyed TCE was added to the tank from the top to form a zone of residual DNAPL saturation across the vertical depth of the tank and small DNAPL pools perched at the low permeable lens and at the bottom. The tank was flushed with $\mathrm{KMnO}_{4}$, and once the pore plugging caused the difficulty in flow, chemical addition was pumped through the tank. The $\mathrm{MnO}_{2}$ precipitates would be dissolved slowly and once the whole flow tank was clean of precipitates, the $\mathrm{KMnO}_{4}$ flushing would resume to continue the oxidation of the remaining DNAPL in the tank. This process was repeated until all the DNAPLs in the flow tank were cleaned up. Both citric acid and oxalic acid was used to dissolve the $\mathrm{MnO}_{2}$ precipitates. EDTA was not used in the flow tank experiment, due to the concern of environmental regulation, even though it has the fast dissolution rate in the batch experiment. Effluent samples were taken three times a day and were analyzed. Images of the column were taken with a digital camera to monitor the development of the growing zone of $\mathrm{MnO}_{2}$ precipitation and the removal of the DNAPLs. 
The results from these experiments indicate that the permanganate oxidation flushing of the source zone combined with the organic acid dissolution flushing can lead to complete removal of the DNAPL of chlorinated solvent contamination, under the condition of the experiment. Further experiments of a larger scale will help to determine the feasibility in successfully clean up the actual contaminated sites.

(3) 3-D flow tank experiment: A large-scale ( $\mathrm{L} \times \mathrm{W} \times \mathrm{D}=180 \times 60 \times 90 \mathrm{~cm}) 3$-D flow sand tank experiment has been conducted to verify the hydraulic impact of solid reaction product $\left(\mathrm{MnO}_{2}\right)$ of permanganate oxidation on the flux of water through the zone of TCE contamination $(\mathrm{L} \mathrm{x} \mathrm{W} \mathrm{x}$ $\mathrm{H}=30 \times 16 \times 36 \mathrm{~cm}$ ) installed in the upstream site within the sandy aquifer.

The tank experiment utilizes chemical, electrical, and spectrometric monitoring schemes to produce resistivity (i.e., reciprocal of conductivity), permanganate concentration, chloride concentration, and TCE concentration data for water samples and TCE concentration data for solid samples. It also uses optical monitoring system to generate photo data that allow the visual observations of the zones of non-reacted permanganate solution (purple), oxidation reaction and $\mathrm{MnO}_{2}$ deposits (black), and non-reacted TCE (colorless) within the aquifer and on the surface layers of the excavated aquifer.

The tank is instrumented with a line of multi-level samplers $(\mathrm{n}=5)$ and glass tubes $(16 \mathrm{~mm}$ diameter; $n=9$ ) down gradient from the emplaced TCE source. Optical data are taken using a color CCD camera (7 mm-diameter) that is lowered down to the bottom of the glass tubes and gradually raised up, while recording. The images recorded using a video recorder is then converted to digital data sets, which can be presented as snap shots. Electrical monitoring schemes depend on measuring the change in the electrical resistivity over time using a Wenner array configuration involving a set of vertically emplaced electrodes. Permanganate, chloride, and TCE concentrations are analyzed using UV-visible spectrophotometer, chloridometer, and GC, respectively.

Figure 1 shows cross-sectional view of the tank with TCE contaminated zone and sampling/monitoring schemes. Visual observations on left side show from the top (1) non-reacted permanganate solution over the source zone (purple), (2) $\mathrm{MnO}_{2}$ deposits in TCE plume (black), (3) non-reacted dissolved TCE plume (colorless), and (4) some $\mathrm{MnO}_{2}$ precipitates (black) under TCE plume.

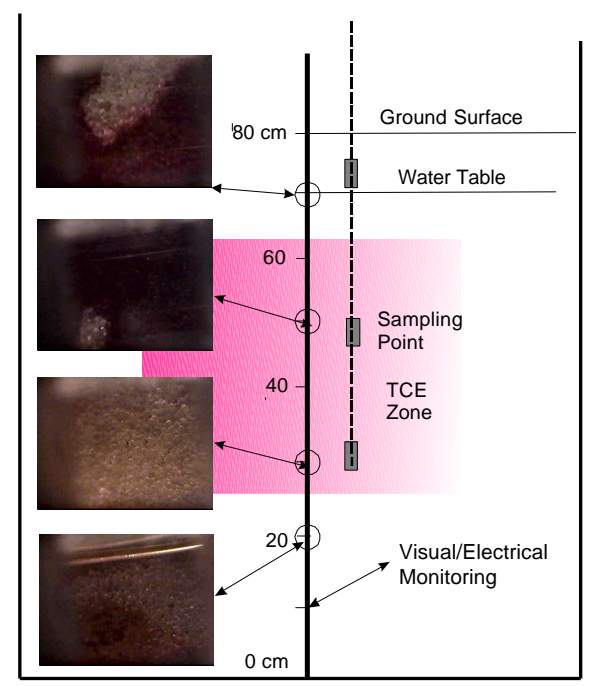

Fig. 1 Cross-sectional view of the tank and sampling/monitoring schemes. 
Figure 2 shows the changes of (a) permanganate and (b) TCE contours along the tank crosssection over time. Contours representing the zones of higher TCE concentration are generally in keeping with the contours representing the zones of lower permanganate concentrations indicating the destruction of TCE by permanganate oxidation.

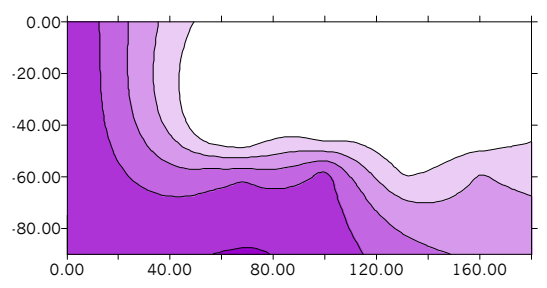

(a) Early Stage (2 days)

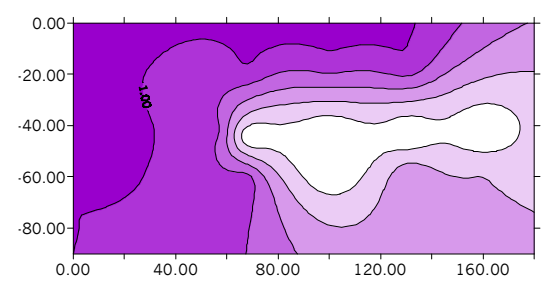

(b) Intermediate Stage (37 days)

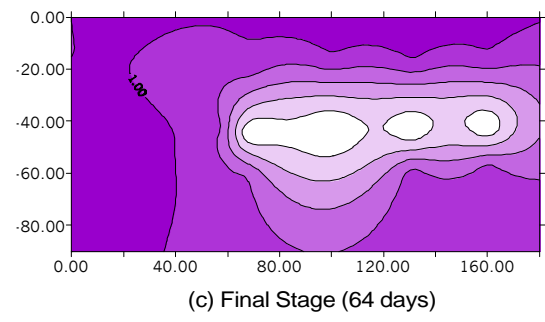

Fig. 2a permanganate contours along the tank cross section over time.

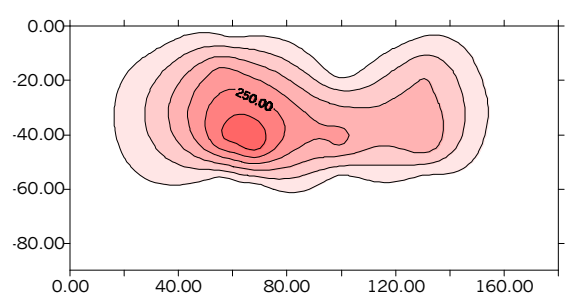

(a) Early Stage (2 days)

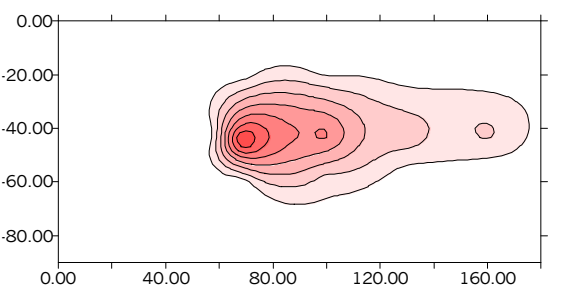

(b) Intermediate Stage (37 days)

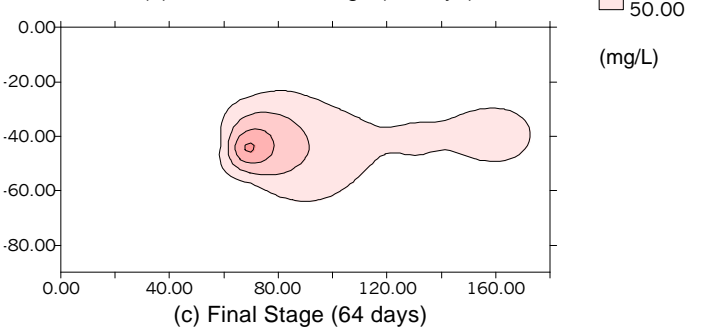

Fig. 2b TCE contours along the tank cross section over time.

Figure 3 shows the changes of (a) resistivity and (b) chloride contours along the tank crosssection over time. Zones of lower resistivity generally coincide with zones of higher chloride concentrations. The zone of elevated chloride also matched the zones of high TCE (Fig. 2b). These observations indicate that the release of chloride from the TCE by permanganate oxidation decreased the resistivity in water. 


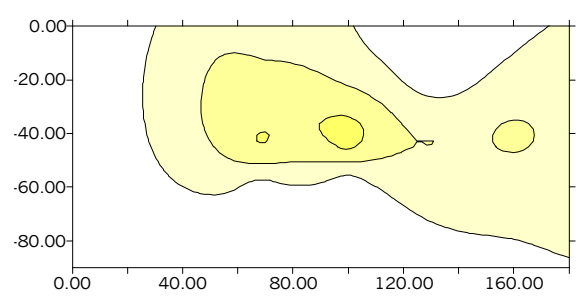

(a) Early Stage (2 days)

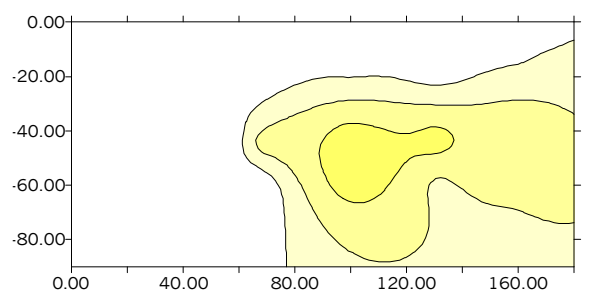

(b) Intermediate Stage (37 days)

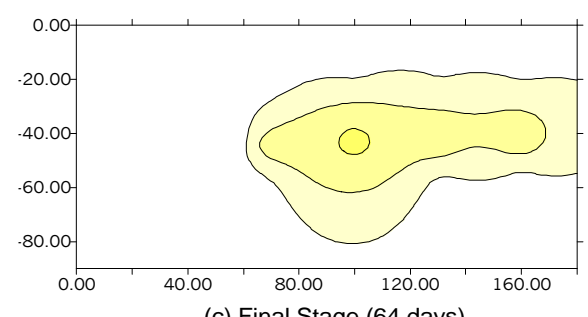

(c) Final Stage (64 days)

Fig. 3a Resistivity contours along the tank cross section over time.

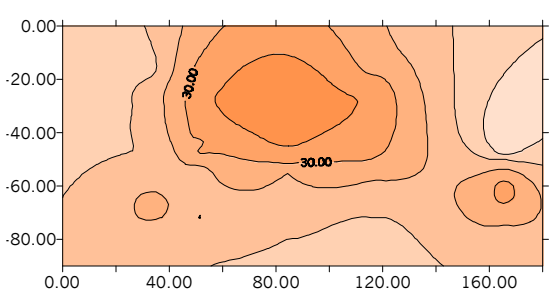

(a) Early Stage (3 days)

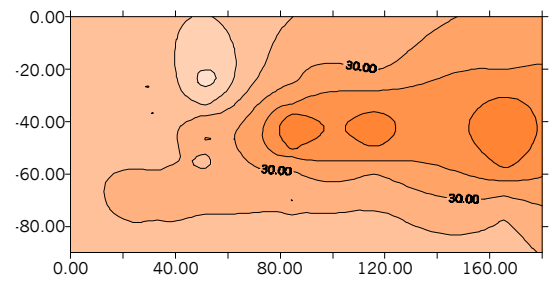

(b) Intermediate Stage (35 days)

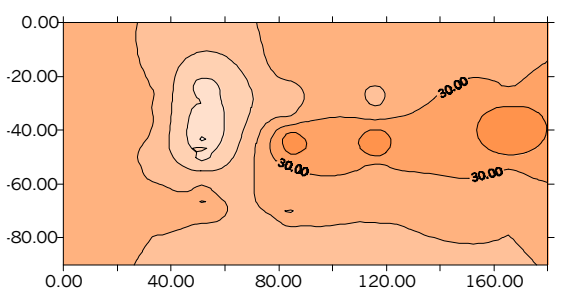

(c) Final Stage (64 days)

Fig. 3b Chloride contours along the tank cross section over time.

Figure 4 shows the images of the excavated surfaces of the sandy aquifer at (a) $15 \mathrm{~cm}$ and (b) 30 $\mathrm{cm}$ depths. The presence of black $\mathrm{MnO}_{2}$ precipitates in the upstream site indicates active permanganate oxidation in the TCE source zone. Black $\mathrm{MnO}_{2}$ precipitates are found along the boundary of the downstream TCE flume (Fig.4a,b).

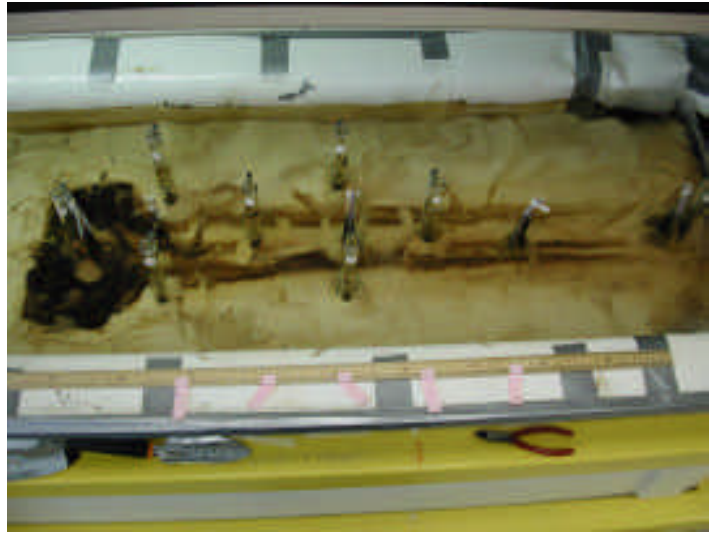

Fig. 4a Excavated surface at $15 \mathrm{~cm}$ depth.

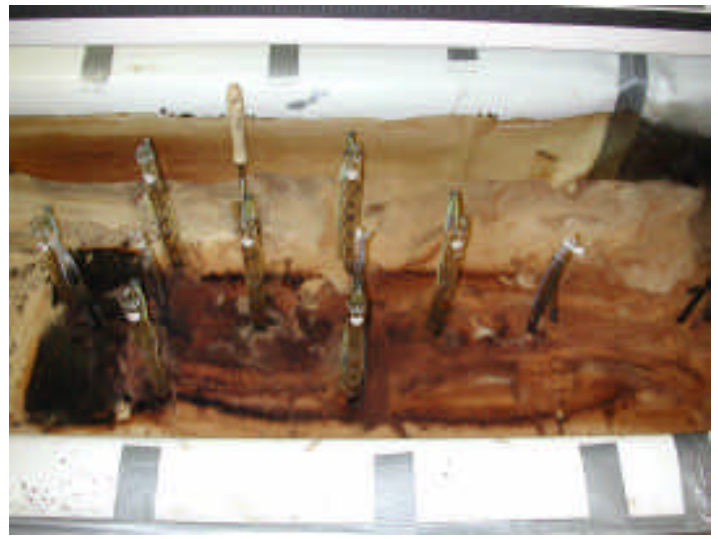

Fig. 4b Excavated surface at $30 \mathrm{~cm}$ depth.

Down-hole optical data (Fig.1) and the TCE and permanganate contours at intermediate and final stages (Figs.2, 3) show that permanganate is moving above and below the dissolved TCE plume. Similar pattern is identified from the optical data of the excavated aquifer surfaces (Fig.4). These 
data indicate that the dissolved TCE plume is surrounded by the $\mathrm{MnO}_{2}$ precipitates, which could lower the hydraulic conductivity of the medium and reduce the efficiency of the reaction.

\section{Planned Activities}

During the next year, our group will continue working on the various fronts of current research. A thorough understanding of the dissolution kinetics will help to find the best chemical additions to control or eliminate the precipitate which causes the poor efficiency of the source zone flooding scheme. Specific tasks are: (1) to determine the dissolution rate during the reaction of manganese solid with different chemical additions by performing a series batch experiment; (2) to determine the solubility of the manganese oxide present with different chemical additions; (3) to perform 2$\mathrm{D}$ and 3-D flow tank experiment to test the best chemical addition, which have determined based on task (1) and (2); (4) to further test the permanganate reactive barrier system in a larger, more realistic flow tank experiment.

\section{Information Access}

Zhang, H., and Schwartz, F. W., 2000, Simulation of oxidative treatment of chlorinated compounds by permanganate. In Chemical Oxidation and Reactive Barriers: Remediation of Chlorinated and Recalcitrant Compounds, Wickramanayake, G. B., Gavaskar, A. R., and Chen, A. S. C. (Editors), 2000, Bettelle Press, Columbus, OH. p 1-8.

Li, X. David and Schwartz, Frank W., 2000, Efficiency problems related to permanganate oxidation schemes. In Chemical Oxidation and Reactive Barriers: Remediation of Chlorinated and Recalcitrant Compounds, Wickramanayake, G. B., Gavaskar, A. R., and Chen, A. S. C. (Editors), 2000, Bettelle Press, Columbus, OH. p 41-48.

Seol, Y. and Schwartz, Frank W., 2000, Phase-transfer catalysis on the oxidation of trichloroethylene by permanganate. In Chemical Oxidation and Reactive Barriers: Remediation of Chlorinated and Recalcitrant Compounds, Wickramanayake, G. B., Gavaskar, A. R., and Chen, A. S. C. (Editors), 2000, Bettelle Press, Columbus, OH. p 17-24.

Li, X. David and Schwartz, Frank W., 2000, Chemical changes during the in situ permanganate treatment of chlorinated solvents in the subsurface, Geological Society of American annual meeting, Nov. 2000, Reno, NV.

Li, X. David and Schwartz, Frank W., 2001, DNAPL source zone remediation by permanganate oxidation, DOE SCFA Midyear review conference, March 2001, Atlanta, GA.

Li, X. David and Schwartz, Frank W., 2001, Experimental test of a newly designed permanganate reactive barrier system for chlorinated solvents remediation. American chemical society annual meeting, April 2001, San Diego, CA.

Seol, Y., Schwartz, Frank W., Lee, S., 2001, Oxidation of binary DNAPL mixtures using potassium permanganate with a phase transfer catalyst. Ground Water Monitoring and Remediation, Spring 2001, p124-132.

Li, X. David and Schwartz, Frank W., 2001, Permanganate Oxidation for the Remediation of Source Zone DNAPL and Contaminant Plume, in Innovative strategies for the remediation of chlorinated solvents and DNAPLs in the subsurface, Henry, S. and Warner, S. (Editors), ACS 
press, Washington, DC. (In press). 